\title{
Weak Disorder Expansion of Liapunov Exponents in a Degenerate Case
}

\author{
N. Zanon ${ }^{1}$ and B. Derrida ${ }^{2}$
}

Received June 24, 1987; revision received October 8, 1987

\begin{abstract}
It is shown how the weak disorder expansion of the Liapunov exponents of a product of random matrices can be derived when the unperturbed matrices have two degenerate eigenvalues. The general expression of the Liapunov exponents at the lowest nontrivial order in disorder is given.
\end{abstract}

KEY WORDS: Disordered systems; products of random matrices; Liapunov exponents; dynamic systems; one-dimensional localization.

\section{INTRODUCTION}

The analytic calculation of the Liapunov exponents of products of random matrices is a problem that arises often in the physics of disordered systems $^{(1)}$ (random magnets, diffusion, or localization in random media: see Refs. 2 and 3 and references therein) and in the study of dynamical systems. ${ }^{(4-6)}$ It is also a very important problem in the theory of probability. ${ }^{(7,15-18)}$

Apart from a few special cases which can be solved exactly, ${ }^{(8,9)}$ there does not exist any general method for calculating analytically the Liapunov exponents of products of random matrices. At present, one can only hope to develop a perturbation method that gives the weak disorder expansion of the Liapunov exponents. The problem can then be formulated as follows: Consider a product of $N$ random $n \times n$ matrices $M_{\alpha}$ :

$$
\operatorname{Prod}=\prod_{\alpha=1}^{N} M_{\alpha}
$$

\footnotetext{
${ }^{1}$ Service de Physique du Solide et de Résonance Magnétique, CEN-Saclay, 91191 Gif-surYvette, France.

${ }^{2}$ Service de Physique Théorique, CEN-Saclay, 91191 Gif-sur-Yvette, France.
} 
where

$$
M_{\alpha}=A+\mu B_{\alpha}
$$

The matrix $A$ is fixed (i.e., does not depend on $\alpha$ ), the matrices $B_{\alpha}$ are random and independent, and one wants to calculate the expansion in powers of $\mu$ of the Liapunov exponents. Such an expansion has recently been obtained in the nondegenerate case, ${ }^{(3)}$ i.e., when the matrix $A$ has all its eigenvalues with different moduli

$$
A=\left(\begin{array}{lll}
\lambda_{1} & & 0 \\
& \ddots & \\
0 & & \lambda_{n}
\end{array}\right)
$$

with

$$
\left|\lambda_{1}\right|>\left|\lambda_{2}\right| \cdots>\left|\lambda_{n}\right|
$$

and the weak disorder expansion of the sum of the first $p$ Liapunov exponents up to the fourth order in $\mu$ is

$$
\begin{aligned}
\gamma_{1}+\gamma_{2}+\cdots+\gamma_{p}= & \sum_{j=1}^{p} \log \lambda_{j}-\frac{\mu^{2}}{2} \sum_{i=1}^{p} \sum_{j=1}^{p} \frac{\left\langle B^{i j} B^{j i}\right\rangle}{\lambda_{i} \lambda_{j}} \\
& +\frac{\mu^{3}}{3} \sum_{i=1}^{p} \sum_{j=1}^{p} \sum_{k=1}^{p} \frac{\left\langle B^{i j} B^{j k} B^{k i}\right\rangle}{\lambda_{i} \lambda_{j} \lambda_{k}} \\
& -\frac{\mu^{4}}{4} \sum_{i=1}^{p} \sum_{j=1}^{p} \sum_{k=1}^{p} \sum_{l=1}^{p} \frac{\left\langle B^{i j} B^{j k} B^{k l} B^{l i}\right\rangle}{\lambda_{i} \lambda_{j} \lambda_{k} \lambda_{l}} \\
& -\frac{\mu^{4}}{2} \sum_{i=1}^{p} \sum_{j=1}^{p} \sum_{r>p}^{n} \sum_{s>p}^{n} \frac{\left\langle B^{i r} B^{j s}\right\rangle\left\langle B^{r j} B^{s i}\right\rangle}{\lambda_{i} \lambda_{j}\left(\lambda_{i} \lambda_{j}-\lambda_{r} \lambda_{s}\right)} \\
& +\mu^{4} \sum_{i=1}^{p} \sum_{j=1}^{p} \sum_{k=1}^{p} \sum_{r>p}^{n} \frac{\left\langle B^{i r} B^{k i}\right\rangle\left\langle B^{r j} B^{j k}\right\rangle}{\lambda_{i} \lambda_{j} \lambda_{k}\left(\lambda_{k}-\lambda_{r}\right)}
\end{aligned}
$$

where $\langle\cdots\rangle$ means the average over disorder, i.e.,

$$
\left\langle B^{i j} B^{r s}\right\rangle=\lim _{N \rightarrow \infty} \frac{1}{N} \sum_{\alpha=1}^{N} B_{\alpha}^{i j} B_{\alpha}^{r s}
$$

One has assumed that $\left\langle B_{\alpha}\right\rangle=0$.

The next question, of course, is whether condition (4) could be entirely relaxed and if one could extend the result (5) to more general cases. In particular this would include cases where several eigenvalues of the matrix $A$ 
are degenerate, the matrix $A$ being diagonalizable or not, and where some eigenvalues have the same modulus. This final goal has not yet been reached.

In the present paper, we describe one step toward the solution of this question. We consider the case of a diagonalizable matrix $A$ with only two degenerate eigenvalues and we calculate the Liapunov exponents up to order $\mu^{2}$.

In Section 2 we describe our main calculation, which concerns the case of $2 \times 2$ matrices, where

where

$$
\operatorname{Prod}=\prod_{\alpha}\left(A+\mu B_{\alpha}\right)
$$

$$
A=\left(\begin{array}{cc}
1+\mu^{2} \varphi_{1} & \mu^{2} \varphi_{2} \\
\mu^{2} \varphi_{3} & 1+\mu^{2} \varphi_{4}
\end{array}\right)
$$

and

$$
B_{\alpha}=\left(\begin{array}{ll}
a_{\alpha} & b_{\alpha} \\
c_{\alpha} & d_{\alpha}
\end{array}\right)
$$

The matrix $A$ does not change with $\alpha$ and the matrices $B_{x}$ are independently distributed. We assume that

$$
\left\langle a_{\alpha}\right\rangle=\left\langle b_{\alpha}\right\rangle=\left\langle c_{\alpha}\right\rangle=\left\langle d_{\alpha}\right\rangle=0
$$

and arbitrary correlations between the elements of $B_{\alpha}$. For simplicity we will consider that the elements of the matrix $B_{x}$ are real. The constants $\varphi_{i}$ in (8) allow us to go continuously from the degenerate case to the nondegenerate case.

We will show that the largest Liapunov exponent $\gamma_{1}$ of the product (7) is given by

$$
\gamma_{1}=\frac{\mu^{2}}{2}\left[\int_{-\infty}^{+\infty} P_{0}(R) G(R) d R\right] /\left[\int_{-\infty}^{+\infty} P_{0}(R) d R\right]+O\left(\mu^{3}\right)
$$

where $P_{0}(R)$ and $G(R)$ are given by the correlations between the elements of the matrices $B_{\alpha}$ :

$$
\begin{aligned}
G(R)= & \frac{1}{\left(R^{2}+\theta^{2}\right)^{2}}\left(\theta^{2}-R^{2}\right)\left[\left\langle a_{\alpha}^{2}\right\rangle R^{2}+2\left\langle a_{\alpha} b_{\alpha}\right\rangle R+\left\langle b_{\alpha}^{2}\right\rangle\right. \\
& \left.-\left\langle c_{\alpha}^{2}\right\rangle R^{2} \theta^{2}-2\left\langle c_{\alpha} d_{\alpha}\right\rangle R \theta^{2}-\left\langle d_{\alpha}^{2}\right\rangle \theta^{2}\right] \\
& -\frac{4 \theta^{2}}{\left(R^{2}+\theta^{2}\right)^{2}}\left[\left\langle a_{\alpha} c_{\alpha}\right\rangle R^{3}+\left\langle b_{\alpha} c_{\alpha}\right\rangle R^{2}+\left\langle a_{\alpha} d_{\alpha}\right\rangle R^{2}+\left\langle b_{\alpha} d_{\alpha}\right\rangle R\right] \\
& +\frac{2}{R^{2}+\theta^{2}}\left(\varphi_{1} R^{2}+\varphi_{2} R+\varphi_{3} R \theta^{2}+\varphi_{4} \theta^{2}\right)
\end{aligned}
$$




$$
\begin{aligned}
P_{0}(R)= & \int_{R}^{\infty} \frac{d x}{H_{1}(x)} \exp \left[\int_{R}^{x} d y \frac{H_{2}(y)}{H_{1}(y)}\right] \\
& +\lim _{\Lambda \rightarrow \infty}\left(\exp \left[\int_{-\Lambda}^{+A} d y \frac{H_{2}(y)}{H_{1}(y)}\right]\right) \int_{-\infty}^{R} \frac{d x}{H_{1}(x)} \exp \left[\int_{R}^{x} d y \frac{H_{2}(y)}{H_{1}(y)}\right]
\end{aligned}
$$

where

$$
\begin{aligned}
H_{1}(x)= & \frac{1}{2}\left\langle\left(c_{\alpha} x^{2}+d_{\alpha} x-a_{\alpha} x-b_{\alpha}\right)^{2}\right\rangle \\
H_{2}(x)= & \varphi_{3} x^{2}+\varphi_{4} x-\varphi_{1} x-\varphi_{2} \\
& +\left\langle\left(c_{\alpha} x-a_{\alpha}\right)\left[c_{\alpha} x^{2}+\left(d_{\alpha}-a_{\alpha}\right) x-b_{\alpha}\right]\right\rangle
\end{aligned}
$$

The expression (11) depends on $G(R)$ and therefore depends in principle on the parameter $\theta$ which appears in (12). It turns out that when one performs the integral (11), the dependence on $\theta$ disappears as long as $\theta \neq 0$ and $\theta \neq \infty$. since

Once $\gamma_{1}$ is known, the second Liapunov exponent $\gamma_{2}$ is easy to obtain,

$$
\gamma_{1}+\gamma_{2}=\left\langle\log \left[\operatorname{det}\left(A+\mu B_{\alpha}\right)\right]\right\rangle
$$

and therefore

$$
\gamma_{2}=\mu^{2}\left[\varphi_{1}+\varphi_{4}-\frac{1}{2}\left\langle a_{\alpha}^{2}\right\rangle-\frac{1}{2}\left\langle d_{\alpha}^{2}\right\rangle-\left\langle b_{\alpha} c_{\alpha}\right\rangle\right]-\gamma_{1}+O\left(\mu^{3}\right)
$$

In Section 3 we discuss two simple examples for which the integral (11) can be done explicitly: one example for which $a_{\alpha}=0, d_{\alpha}=0$ for all $\alpha$ and $\left\langle c_{\alpha}^{2}\right\rangle=\left\langle b_{\alpha}^{2}\right\rangle, c_{\alpha}$ and $b_{\alpha}$ being independent, and another example for which $\left\langle a_{\alpha}^{2}\right\rangle=\left\langle b_{\alpha}^{2}\right\rangle=\left\langle c_{\alpha}^{2}\right\rangle=\left\langle d_{\alpha}^{2}\right\rangle$, these four elements being independent.

In Section 4 we extend the results of Section 2 to the case of two degenerate eigenvalues in an $n \times n$ matrix.

\section{THE CASE OF $2 \times 2$ MATRICES}

In this section we calculate $\gamma_{1}$ up to order $\mu^{2}$ for the case where the matrices $A$ and $B$ are given by (8) and (9). Consider the sequence of vectors $V_{\alpha}$ such that

$$
\mathbf{V}_{\alpha+1}=\left(A+\mu B_{\alpha}\right) \mathbf{V}_{\alpha}
$$

If $u_{\alpha}^{(1)}$ and $u_{\alpha}^{(2)}$ are the two components of the vector $\mathbf{V}_{\alpha}$, they satisfy the following recursion relations:

$$
\begin{aligned}
& u_{\alpha+1}^{(1)}=\left(1+\mu a_{\alpha}+\mu^{2} \varphi_{1}\right) u_{\alpha}^{(1)}+\left(\mu b_{\alpha}+\mu^{2} \varphi_{2}\right) u_{\alpha}^{(2)} \\
& u_{\alpha+1}^{(2)}=\left(\mu c_{\alpha}+\mu^{2} \varphi_{3}\right) u_{\alpha}^{(1)}+\left(1+\mu d_{\alpha}+\mu^{2} \varphi_{4}\right) u_{\alpha}^{(2)}
\end{aligned}
$$


The largest Liapunov exponent $\gamma_{1}$ is then given by

$$
\gamma_{1}=\lim _{N \rightarrow \infty} \frac{1}{2 N} \log \left[\frac{\left(u_{N}^{(1)}\right)^{2}+\theta^{2}\left(u_{N}^{(2)}\right)^{2}}{\left(u_{0}^{(1)}\right)^{2}+\theta^{2}\left(u_{0}^{(2)}\right)^{2}}\right]
$$

Expression (20) expresses the fact that the Liapunov exponent $\gamma_{1}$ measures the way the length of the vector $\mathbf{V}_{N}$ increases with $N$. There are several ways of measuring this length [which are represented by the parameter $\theta$ in formula (20)] and all these definitions should give the same result for $\gamma_{1}$.

An often used method $^{(2)}$ to compute the Liapunov exponent $\gamma_{1}$ consists in looking for the stationary probability distribution $P(R)$ of a variable $R_{\alpha}$ which gives the direction of the vector $\mathbf{V}_{\alpha}$ :

$$
R_{\alpha}=u_{\alpha}^{(1)} / u_{\alpha}^{(2)}
$$

The recursion relation on $R_{\alpha}$ is a homographic transformation $T_{\alpha}$ [see Eq. (19)]

$$
R_{\alpha+1}=T_{\alpha}\left(R_{\alpha}\right)=\frac{\left(1+\mu a_{\alpha}+\mu^{2} \varphi_{1}\right) R_{\alpha}+\mu b_{\alpha}+\mu^{2} \varphi_{2}}{\left(\mu c_{\alpha}+\mu^{2} \varphi_{3}\right) R_{\alpha}+1+\mu d_{\alpha}+\mu^{2} \varphi_{4}}
$$

The stationary probability distribution $P(R)$ satisfies the following integral equation:

$$
P(R)=\int P\left(R_{1}\right) d R_{1}\left\langle\delta\left(R-T_{\alpha}\left(R_{1}\right)\right)\right\rangle
$$

where in $(23)$ the $\langle\cdots\rangle$ means the average over $\alpha$, i.e., the average over disorder.

If one knows the solution $P(R)$ of this integral equation, the Liapunov exponent $\gamma_{1}$ is then obtained by

$$
\begin{aligned}
\gamma_{1}= & \frac{1}{2} \int P(R) d R\left\langle\operatorname { l o g } \left\{\left[\left(1+\mu a_{\alpha}+\mu^{2} \varphi_{1}\right) R+\mu b_{\alpha}+\mu^{2} \varphi_{2}\right]^{2}\right.\right. \\
& \left.+\theta^{2}\left[\left(\mu c_{\alpha}+\mu^{2} \varphi_{3}\right) R+1+\mu d_{\alpha}+\mu^{2} \varphi_{4}\right]^{2}\right\} \\
& \left.-\log \left(R^{2}+\theta^{2}\right)\right\rangle / \int P(R) d R
\end{aligned}
$$

[the integral $\int P(R) d R$ in the denominator is necessary in the case that the solution of (23) is not normalized]. This expression (24) is rather easy to understand, since (20) can be written as

$$
\gamma_{1}=\lim _{N \rightarrow \infty} \frac{1}{N} \sum_{\alpha=1}^{N} \frac{1}{2} \log \left[\frac{\left(u_{\alpha+1}^{(1)}\right)^{2}+\theta^{2}\left(u_{\alpha+1}^{(2)}\right)^{2}}{\left(u_{\alpha}^{(1)}\right)^{2}+\theta^{2}\left(u_{\alpha}^{(2)}\right)^{2}}\right]
$$


In principle, if one could solve exactly the integral equation for $P(R)$, then (24) would give $\gamma_{1}$ for all value of $\mu$. In practice, the integral equation (23) is too hard to solve for general $\mu$ and it is only the expansion around $\mu=0$ that can be done.

Let us first determine $P(R)$ in the limit $\mu \rightarrow 0$.

One can rewrite $(23)$ as

$$
P(R)=\left\langle\frac{d T_{\alpha}^{-1}(R)}{d R} P\left(T_{\alpha}^{-1}(R)\right)\right\rangle
$$

where

$$
T_{\alpha}^{-1}(R)=\frac{R\left(1+\mu d_{\alpha}+\mu^{2} \varphi_{4}\right)-\mu b_{\alpha}-\mu^{2} \varphi_{2}}{1+\mu a_{\alpha}+\mu^{2} \varphi_{1}-R\left(\mu c_{\alpha}+\mu^{2} \varphi_{3}\right)}
$$

For $\mu$ small, $T_{\alpha}^{-1}(R)$ is very close to the identity and one has

$$
T_{\alpha}^{-1}(R)=R+\mu E_{\alpha}(R)+\mu^{2} F_{\alpha}(R)+O\left(\mu^{3}\right)
$$

where

$$
\begin{aligned}
E_{\alpha}(R)= & -b_{\alpha}+R\left(d_{\alpha}-a_{\alpha}\right)+R^{2} c_{\alpha} \\
F_{\alpha}(R)= & a_{\alpha} b_{\alpha}-\varphi_{2}+R\left(\varphi_{4}-\varphi_{1}-a_{\alpha} d_{\alpha}-b_{\alpha} c_{\alpha}+a_{\alpha}^{2}\right) \\
& +R^{2}\left(\varphi_{3}+c_{\alpha} d_{\alpha}-2 a_{\alpha} c_{\alpha}\right)+R^{3} c_{\alpha}^{2}
\end{aligned}
$$

If one replaces $T_{\alpha}^{-1}(R)$ by its expansion (28) in (26), one gets

$$
\begin{aligned}
P(R)= & \left\langle\left(1+\mu \frac{d E_{\alpha}}{d R}+\mu^{2} \frac{d F_{\alpha}}{d R}\right) P\left(R+\mu E_{\alpha}+\mu^{2} F_{\alpha}\right)\right\rangle+O\left(\mu^{3}\right) \\
= & P(R)+\mu\left\langle\left(E_{\alpha} P^{\prime}+E_{\alpha}^{\prime} P\right)\right\rangle \\
& +\mu^{2}\left\langle F_{\alpha}^{\prime} P+F_{\alpha} P^{\prime}+E_{\alpha}^{\prime} E_{\alpha} P^{\prime}+\frac{1}{2} E_{\alpha}^{2} P^{\prime \prime}\right\rangle+O\left(\mu^{3}\right)
\end{aligned}
$$

Using (10), one sees that the linear term in $\mu$ vanishes and that the order $\mu^{2}$ of (30) gives a differential equation for $P$ :

$$
H_{2}^{\prime} P+\left[H_{2}+H_{1}^{\prime}\right] P^{\prime}+H_{1} P^{\prime \prime}=O(\mu)
$$

If one considers that $P$ can be expanded,

$$
P(R)=P_{0}(R)+\mu P_{1}(R)+\mu^{2} P_{2}(R)+\cdots
$$

one sees from (33) that $P_{0}$ must satisfy

$$
H_{2}^{\prime} P_{0}+\left(H_{2}+H_{1}^{\prime}\right) P_{0}^{\prime}+H_{1} P_{0}^{\prime \prime}=0
$$


where the two functions $H_{1}$ and $H_{2}$ are given by

$$
H_{1}(R)=\frac{1}{2}\left\langle E_{\alpha}^{2}\right\rangle, \quad H_{2}(R)=\left\langle F_{\alpha}\right\rangle
$$

Looking at (14), (15), (29), and (30), one can easily see that the general solution of Eq. (33) is

$$
\begin{aligned}
P_{0}(R)= & K_{1} \int_{R}^{\infty} \frac{d x}{H_{1}(x)} \exp \left[\int_{R}^{x} d y \frac{H_{2}(y)}{H_{1}(y)}\right] \\
& +K_{2} \int_{-\infty}^{R} \frac{d x}{H_{1}(x)} \exp \left[\int_{R}^{x} d y \frac{H_{2}(y)}{H_{1}(y)}\right]
\end{aligned}
$$

The two constants $K_{1}$ and $K_{2}$ in (37) are the two constants of integration of the differential equation (33).

In order to determine these two constants $K_{1}$ and $K_{2}$ and therefore the probability distribution $P_{0}(R)$, one needs to impose constraints. One of these constraints is clearly the normalization of $P_{0}(R)$, but can be forgotten because of the denominator in (24). The other can be determined by saying that $P_{0}(R)$ has the same behavior for $R \rightarrow+\infty$ and $R \rightarrow-\infty$. This is because $R$ is a variable, which gives the angle of the vector $V_{\alpha}$, and because the direction $R=\infty$ does not play any particular role. Therefore, since, from (37), one can see that

$$
P_{0}(R)=\frac{K_{2}}{R^{2}} \lim _{A \rightarrow \infty} \Lambda^{2} \int_{-\infty}^{+\infty} \frac{d x}{H_{1}(x)} \exp \int_{A}^{x} d y \frac{H_{2}(y)}{H_{1}(y)} \text { for } R \rightarrow+\infty
$$

because $H_{2}(y) / H_{1}(y) \sim 2 / y$ when $y \rightarrow \infty$ [see Eqs. (36), (29), (30)] and

$$
P_{0}(R)=\frac{K_{1}}{R^{2}} \lim _{A^{\prime} \rightarrow \infty} A^{\prime 2} \int_{-\infty}^{+\infty} \frac{d x}{H_{1}(x)} \exp \int_{-A^{\prime}}^{x} d y \frac{H_{2}(y)}{H_{1}(y)} \text { for } R \rightarrow-\infty
$$

where in (38) and (39) the cutoffs $A$ and $A^{\prime}$ are introduced because the integral of $H_{2} / H_{1}$ diverges logarithmically.

The condition that $P_{0}(R)$ has the same behavior at $+\infty$ and $-\infty$ imposes the condition that

$$
K_{2}=K_{1} \lim _{\substack{A \rightarrow \infty \\ A^{\prime} \rightarrow \infty}}\left(\frac{A^{\prime}}{\Lambda}\right)^{2} \exp \int_{-\Lambda^{\prime}}^{\Lambda} d y \frac{H_{2}(y)}{H_{1}(y)}
$$

Choosing, for example, $\Lambda=\Lambda^{\prime}$ gives the expression (13) for $P_{0}$ up to the 
normalization constant. Once $P_{0}(R)$ is known, one can use (24) to compute $\gamma_{1}$. If one expands (24) up to second order in $\mu$, one gets for $\gamma_{1}$

$$
\gamma_{1}=\frac{1}{2} \mu^{2}\left[\int P_{0}(R) G(R) d R\right] / \int P_{0}(R) d R
$$

where $G(R)$ is given by (12).

Since $G(R)$ depends on $\theta$ [see Eq. (12)], it is not obvious that the final result $\gamma$ given by (11) is independent of $\theta$. However, this can be checked already on the expression (24), which can be rewritten as

$$
\begin{aligned}
\gamma_{1}= & \int P(R) d R\left\langle\log \left[\left(\mu c_{\alpha}+\mu^{2} \varphi_{3}\right) R+1+\mu d_{\alpha}+\mu^{2} \varphi_{4}\right]\right\rangle \\
& +\frac{1}{2} \int P(R) d R\left\langle\log \left\{[T(R)]^{2}+\theta^{2}\right\}\right\rangle-\frac{1}{2} \int P(R) d R \log \left(R^{2}+\theta^{2}\right)
\end{aligned}
$$

We see from Eq. (26) that the second integral is equal to the third one in (42), so the $\theta$ dependence disappears in the expression for $\gamma_{1}$. Let us now look at the expression (41). Once we have the expansion for $\mu$ small, from (12), (14), and (15), $G(R)$ can be written as

$$
\begin{aligned}
G(R)= & {\left[2 \varphi_{3} R+2 \varphi_{4}-\left\langle c_{x}^{2}\right\rangle R^{2}-2\left\langle c_{\alpha} d_{\alpha}\right\rangle R-\left\langle d_{\alpha}^{2}\right\rangle\right] } \\
& +\left[H_{1}(R) \frac{\partial^{2} \log \left(R^{2}+\theta^{2}\right)}{\partial R^{2}}\right]+\left\{\left[H_{1}^{\prime}(R)-H_{2}(R)\right] \frac{\partial \log \left(R^{2}+\theta^{2}\right)}{\partial R}\right\}
\end{aligned}
$$

Noticing that

$$
\begin{aligned}
P_{0}(R) & H_{1}(R) \frac{\partial^{2} \log \left(R^{2}+\theta^{2}\right)}{\partial R^{2}} \\
= & \frac{d}{d R}\left[P_{0}(R) H_{1}(R) \frac{\partial}{\partial R} \log \left(R^{2}+\theta^{2}\right)\right] \\
& -\left[\frac{d P}{d R} H_{1}(R)+P_{0}(R) \frac{d H_{1}}{d R}\right] \frac{\partial \log \left(R^{2}+\theta^{2}\right)}{\partial R}
\end{aligned}
$$

and using Eq. (33), that is,

$$
\frac{d}{d R}\left[H_{2}(R) P_{0}(R)+H_{1}(R) \frac{d P_{0}}{d R}\right]=0
$$


we can rewrite $\gamma_{1}$ as

$$
\begin{aligned}
\gamma_{1}= & \frac{\mu^{2}}{2} \int_{-\infty}^{+\infty} d R\left(P_{0}(R)\left[2 \varphi_{3} R+2 \varphi_{4}-\left\langle c_{\alpha}^{2}\right\rangle R^{2}-2\left\langle c_{\alpha} d_{\alpha}\right\rangle R-\left\langle d_{\alpha}^{2}\right\rangle\right]\right. \\
& +\frac{d}{d R}\left\{H_{1}(R) P_{0}(R) \frac{\partial \log \left(R^{2}+\theta^{2}\right)}{\partial R}\right. \\
& \left.\left.-\log \left(R^{2}+\theta^{2}\right)\left[H_{2}(R) P_{0}(R)+H_{1}(R) \frac{d P_{0}}{d R}\right]\right\}\right)
\end{aligned}
$$

Here we see that the $\theta$ dependence of $\gamma_{1}$ appears through a total derivative. Thus, only the behavior in the limit $R \rightarrow \infty$ is important.

If we consider the difference $\gamma_{1}(\theta)-\gamma_{1}(\bar{\theta})$ for two choices $\theta$ and $\bar{\theta}$ of $\theta$ in $(45)$, we get

$$
\begin{aligned}
\gamma_{1}(\theta)-\gamma_{1}(\bar{\theta})= & \frac{\mu^{2}}{2}\left\{H_{1}(R) P_{0}(R) \frac{d}{d R} \log \left(\frac{R^{2}+\theta^{2}}{R^{2}+\bar{\theta}^{2}}\right)\right. \\
& \left.-\log \left(\frac{R^{2}+\theta^{2}}{R^{2}+\bar{\theta}^{2}}\right)\left[H_{2}(R) P_{0}(R)+H_{1}(R) \frac{d P_{0}}{d R}\right]\right\}_{-\infty}^{+\infty}
\end{aligned}
$$

When $R \rightarrow \pm \infty$, the total derivative vanishes, since $P_{0}(R) \sim 1 / R^{2}[$ see $(38)$ and (39)], $H_{1} \sim R^{4}$, and $H_{2} \sim R^{3}$ [see (14) and (15)].

Expression (45) gives a $\gamma_{1}$ independent of $\theta$. However, one cannot set $\theta=0$ or $\theta=\infty$ in this expression because the integral over $R$ becomes divergent either at $R=0$ or $R=\infty$. The reason the above calculation is not valid for $\theta=0$ or $\theta=\infty$ is that in Eq. (19) we considered that $\left|u_{x}^{(1)}\right| \gg \mu$ and $\left|u_{\alpha}^{(2)}\right| \gg \mu$ to expand in powers of $\mu$ and that these conditions are no longer fulfilled if $\theta=0$ or $\theta=\infty$.

Let us remark that the Liapunov exponent measures how the length of a vector increases under the multiplication of the vector by random matrices. There are several equivalent ways of measuring these lengths (corresponding to all the possible choices of $\theta$ ). We have just seen that all choices of $\theta$ give the same $\gamma_{1}$. It would have been possible to make more general choices to measure the length of the vector. For example, one could have used an arbitrary homogeneous function $D$ of the coordinates in (25). Then the expression (45) of $\gamma_{1}$ would read

$$
\begin{aligned}
\gamma_{1}= & \frac{\mu^{2}}{2} \int_{-\infty}^{+\infty} d R\left(P_{0}(R)\left[2 \varphi_{3} R+2 \varphi_{4}-\left\langle c_{\alpha}^{2}\right\rangle R^{2}-2\left\langle c_{\alpha} d_{\alpha}\right\rangle R-\left\langle d_{\alpha}^{2}\right\rangle\right]\right. \\
& +\frac{1}{n} \frac{d}{d R}\left\{H_{1}(R) P_{0}(R) \frac{\partial}{\partial R} \log [D(R)]\right. \\
& \left.\left.-\log [D(R)]\left[H_{2}(R) P_{0}(R)+H_{1}(R) \frac{d P_{0}}{d R}\right]\right\}\right)
\end{aligned}
$$


where

$$
D(R)=\sum_{p=0}^{n} a_{p} R^{2 p}
$$

with arbitrary coefficients $a_{p}>0$. Again $\gamma_{1}$ would not depend on the $a_{p}$ in the same way as it did not depend on $\theta$.

Formula (45) gives the weak disorder expansion of $\gamma_{1}$ up to the second order in $\mu$. It would be possible to generalize it to higher orders. By expanding $T_{\alpha}^{-1}(R)$ [see Eq. (28)] and $P(R)$ [see Eq. (34)] to higher orders in $\mu$ and by replacing $T_{\alpha}^{-1}$ and $P$ by their expansions in (26), we get a hierarchy of equations corresponding to each order $\mu$. The order $\mu^{2}$ gives for $P_{0}$

$$
\frac{d}{d R}\left(\left\langle F_{\alpha}\right\rangle P_{0}+\left\langle\frac{E_{\alpha}^{2}}{2}\right\rangle \frac{d P_{0}}{d R}\right)=0
$$

which is Eq. (33).

The orders $\mu^{3}$ and $\mu^{4}$ give for $P_{1}$ and $P_{2}$, respectively,

$$
\begin{aligned}
& \frac{d}{d R}\left(\left\langle F_{\alpha}\right\rangle P_{1}+\frac{\left\langle E_{\alpha}^{2}\right\rangle}{2} \frac{d P_{1}}{d R}\right) \\
& =-\frac{d}{d R}\left(\left\langle I_{\alpha}\right\rangle P_{0}+\left\langle E_{\alpha} F_{\alpha}\right\rangle \frac{d P_{0}}{d R}+\frac{\left\langle E_{\alpha}^{3}\right\rangle}{6} \frac{d^{2} P_{0}}{d R^{2}}\right) \\
& \frac{d}{d R}\left(\left\langle F_{\alpha}\right\rangle P_{2}+\frac{\left\langle E_{\alpha}^{2}\right\rangle}{2} \frac{d P_{2}}{d R}\right) \\
& =-\frac{d}{d R}\left(\left\langle I_{\alpha}\right\rangle P_{1}+\left\langle E_{\alpha} F_{\alpha}\right\rangle \frac{d P_{1}}{d R}+\frac{\left\langle E_{\alpha}^{3}\right\rangle}{6} \frac{d^{2} P_{1}}{d R^{2}}\right) \\
& \quad-\frac{d}{d R}\left(\left\langle J_{\alpha}\right\rangle P_{0}+\left\langle E_{\alpha} I_{\alpha}+\frac{F_{\alpha}^{2}}{2}\right\rangle \frac{d P_{0}}{d R}+\frac{\left\langle E_{\alpha}^{2} F_{\alpha}\right\rangle}{2} \frac{d^{2} P_{0}}{d R^{2}}+\frac{\left\langle E_{\alpha}^{4}\right\rangle}{4 !} \frac{d^{3} P_{0}}{d R^{3}}\right)
\end{aligned}
$$

where $I_{\alpha}$ and $J_{\alpha}$ are the next terms in the expansion (28):

$$
T_{\alpha}^{-1}(R)=R+\mu E_{\alpha}+\mu^{2} F_{\alpha}+\mu^{3} I_{\alpha}+\mu^{4} J_{\alpha}+O\left(\mu^{5}\right)
$$

Knowing $P_{0}$, we can in principle determine $P_{1}$ from (49) and then $P_{2}$ from (50) and by this method get higher orders in the expansion of the Liapunov exponents.

Lastly let us notice that the expression (13) of $P_{0}(R)$ could be simplified by using instead of $R$ an angular variable $\psi(0<\psi<2 \pi)$,

$$
R=\operatorname{tg}(\psi / 2)
$$


Then, defining a density $Q(\psi)$ by

$$
Q(\psi)=P_{0}\left(\operatorname{tg} \frac{\psi}{2}\right) \frac{1}{2}\left(1+\operatorname{tg}^{2} \frac{\psi}{2}\right)
$$

one would get

$$
\begin{aligned}
Q(\psi)= & \frac{1}{2}\left(1+\operatorname{tg}^{2} \frac{\psi}{2}\right) \int_{\psi}^{\psi+2 \pi} d \varphi\left\{\exp \left[\int_{\psi}^{\varphi} d \alpha \frac{H_{2}\left(\operatorname{tg} \frac{1}{2} \alpha\right)}{H_{1}\left(\operatorname{tg} \frac{1}{2} \alpha\right)} \frac{1}{2}\left(1+\operatorname{tg}^{2} \frac{\alpha}{2}\right)\right]\right. \\
& \left.\times\left[H_{1}\left(\operatorname{tg} \frac{1}{2} \varphi\right)\right]^{-1}\right\}
\end{aligned}
$$

This expression is more symmetric than (13), since all directions $\psi$ play a similar role [the special role of the direction $R=\infty$ in (13) has disappeared].

\section{EXAMPLES}

In order to illustrate the calculations presented in Section 2, we now study two simple examples. The first example is a case where the matrix $A$ is unity $\left(\varphi_{i}=0\right)$ and the matrices $B$ have only off-diagonal elements, which are random and independent

$$
a_{\alpha}=0 ; \quad d_{\alpha}=0 ; \quad\left\langle b_{\alpha} c_{\alpha}\right\rangle=0 ; \quad\left\langle b_{\alpha}^{2}\right\rangle=\left\langle c_{\alpha}^{2}\right\rangle
$$

The expression (13) for $P_{0}$ becomes

$$
P_{0}(R)=\frac{\text { cste }}{\left(1+R^{4}\right)^{1 / 2}}
$$

and then $\gamma_{1}$ is given by [see Eqs. (11) and (12)]

$$
\gamma_{1}=\frac{\left\langle c_{\alpha}^{2}\right\rangle}{2} \mu^{2} \int_{-\infty}^{+\infty} \frac{d R}{\left(1+R^{4}\right)^{1 / 2}} \frac{\left(R^{2}-\theta^{2}\right)\left(1-R^{2} \theta^{2}\right)}{\left(R^{2}+\theta^{2}\right)^{2}} / \int_{-\infty}^{+\infty} \frac{d R}{\left(1+R^{4}\right)^{1 / 2}}+O\left(\mu^{3}\right)
$$

One can easily check again for this example that $\gamma_{1}$ is independent of $\theta$ (if $\theta \neq 0$ and $\theta \neq \infty): \gamma_{1}$ can be written as

$$
\begin{aligned}
\gamma_{1}= & \frac{\mu^{2}}{2}\left\langle c_{\alpha}^{2}\right\rangle \int_{-\infty}^{+\infty} d R\left[-\frac{R^{2}}{\left(1+R^{4}\right)^{1 / 2}}+\frac{d}{d R}\left(\frac{R\left(1+R^{4}\right)^{1 / 2}}{R^{2}+\theta^{2}}\right)\right] \\
& \int_{-\infty}^{+\infty} d R \frac{1}{\left(1+R^{4}\right)^{1 / 2}}
\end{aligned}
$$


Thus, the difference $\gamma_{1}(\theta)-\gamma_{1}(\bar{\theta})$ vanishes:

$$
\begin{aligned}
\gamma_{1}(\theta)-\gamma_{1}(\bar{\theta})= & \frac{\mu^{2}}{2}\left\langle c_{\alpha}^{2}\right\rangle\left[R\left(1+R^{4}\right)^{1 / 2}\left(\frac{1}{R^{2}+\theta^{2}}-\frac{1}{R^{2}+\bar{\theta}^{2}}\right)\right]_{-\infty}^{+\infty} / \\
& \int_{-\infty}^{+\infty} \frac{d R}{\left(1+R^{4}\right)^{1 / 2}}=0
\end{aligned}
$$

Since $\gamma_{1}$ does not depend on $\theta$, we can choose $\theta=1$ :

$$
\gamma_{1}=-\frac{\left\langle c^{2}\right\rangle}{2} \mu^{2} \int_{-\infty}^{+\infty} \frac{d R}{\left(1+R^{4}\right)^{1 / 2}} \frac{\left(R^{2}-1\right)^{2}}{\left(R^{2}+1\right)^{2}} / \int_{-\infty}^{+\infty} \frac{d R}{\left(1+R^{4}\right)^{1 / 2}}
$$

Then the integrals can be expressed in terms of elliptic integrals, ${ }^{(10)}$

$$
\begin{gathered}
\int_{-\infty}^{+\infty} \frac{d R}{\left(1+R^{4}\right)^{1 / 2}}=F\left(\pi, \frac{\sqrt{2}}{2}\right) \\
\int_{-\infty}^{+\infty} \frac{d R}{\left(1+R^{4}\right)^{1 / 2}} \frac{\left(R^{2}-1\right)^{2}}{\left(R^{2}+1\right)^{2}}=2 E\left(\pi, \frac{\sqrt{2}}{2}\right)-F\left(\pi, \frac{\sqrt{2}}{2}\right)
\end{gathered}
$$

Thus,

$$
\gamma_{1}=\left\langle c_{\alpha}^{2}\right\rangle \mu^{2} \cdot 0.1142 \ldots
$$

This result was already obtained in the case of a one-dimensional localization problem. ${ }^{(6,19,20)}$

In the second example, matrix $A$ is again unity $\left(\varphi_{i}=0\right)$, and the matrices $B$ have all their elements independent and Gaussian with the same width,

$$
\left\langle a_{\alpha}^{2}\right\rangle=\left\langle b_{\alpha}^{2}\right\rangle=\left\langle c_{\alpha}^{2}\right\rangle=\left\langle d_{\alpha}^{2}\right\rangle
$$

The expression (13) for $P_{0}$ becomes

$$
P_{0}(R)=\frac{\text { cste }}{1+R^{2}}
$$

Since in that case $G(R)$ is

$$
G(R)=\left\langle a_{\alpha}^{2}\right\rangle \frac{\left(1-\theta^{2}\right)\left(\theta^{2}-R^{2}\right)\left(R^{2}+1\right)}{\left(R^{2}+\theta^{2}\right)^{2}}
$$

one finds

$$
\gamma_{1}=0+O\left(\mu^{3}\right)
$$


This result can be checked, because this example is soluble for any value of $\mu{ }^{(8,11)}$ The probability distribution of the matrices $B_{\alpha}$ is a function of $\operatorname{tr}^{t} B_{\alpha} \cdot B_{\alpha}$ only and therefore is rotationally invariant. So is the probability distribution of the matrices $M_{\alpha}$ and of the vector $v_{\alpha}$. Therefore $P(R)=\operatorname{cste} /\left(1+R^{2}\right)$ is the exact solution of $(23)$.

If we expand the expression

$$
\log \left\{\left[\left(1+\mu a_{\alpha}\right) R+\mu b_{\alpha}\right]^{2}+\theta^{2}\left(\mu c_{\alpha} R+1+\mu d_{\alpha}\right)^{2}\right\}
$$

in $\mu$, we can use (24) to get the expansion of $\gamma_{1}$ up to any order in $\mu$.

Let us notice with Newman ${ }^{(8,11)}$ that the probability distribution of $\left(M_{\alpha} \mathbf{V} \cdot M_{\alpha} \mathbf{V}\right) / \mathbf{V} \cdot \mathbf{V}$ does not depend on the choice of $\mathbf{V}$, because of the rotational invariance of the distribution probability of ${ }^{t} M_{\alpha} M_{\alpha}$. Then it is simpler to consider

$$
\gamma_{1}=\frac{1}{2}\left\langle\log \left(M_{\alpha} \mathbf{W} \cdot M_{\alpha} \mathbf{W}\right)\right\rangle
$$

where

$$
\mathbf{W}=\left(\begin{array}{l}
1 \\
0
\end{array}\right)
$$

We get

$$
\gamma_{1}=\left\langle\frac{1}{2} \log \left[\left(1+\mu a_{\alpha}\right)^{2}+\left(\mu c_{\alpha}\right)^{2}\right]\right\rangle
$$

Since $a_{\alpha}$ and $c_{\alpha}$ are Gaussian variables with zero means and $\left\langle a_{x}^{2}\right\rangle=\left\langle c_{\alpha}^{2}\right\rangle=1$, one can reduce the calculation of the average (71) to the calculation of Gaussian integrals using

$$
\langle\log x\rangle=\int_{0}^{\infty} \frac{e^{-t}-\left\langle e^{-t x}\right\rangle}{t} d t
$$

and one gets

$$
\gamma_{1}=\frac{1}{2} \int_{1 /\left(2 \mu^{2}\right)}^{\infty} \frac{e^{-u}}{u} d u
$$

This exact expression for $\gamma_{1}$ shows that the expansion in powers of $\mu$ is zero at all orders in $\mu$ and this agrees with (67). 


\section{TWO DEGENERATE EIGENVALUES IN AN $n \times n$ MATRIX}

In this section, we extend the results of Section 2 to the case of an $n \times n$ matrix where the $p$ th and $(p+1)$ th eigenvalues are degenerate

$$
A=\left(\begin{array}{lll}
\lambda_{1} & & 0 \\
& \ddots & \\
0 & & \lambda_{n}
\end{array}\right)
$$

with

$$
\lambda_{1}>\lambda_{2}>\cdots>\lambda_{p}=\lambda_{p+1}>\cdots>\lambda_{n}
$$

The expansion (5) gives us the weak disorder expansion of any sum $\sum_{j=1}^{m} \gamma_{j}, m \neq p$.

We shall show that $\gamma_{p}$ can also be calculated by the same method as $\gamma_{1}$ in Section 2: The result will be

$$
\gamma_{p}=\frac{1}{2} \mu^{2} \int P_{0}(R) G(R) d R / \int P_{0}(R) d R
$$

where $P_{0}(R)$ and $G(R)$ are still given by formulas (13) and (12) with the $\varphi_{i}$ and $a_{\alpha}, b_{\alpha}, c_{\alpha}, d_{\alpha}$ being replaced by the $\tilde{\varphi}_{i}$ and $\tilde{a}_{\alpha}, \tilde{b}_{\alpha}, \tilde{c}_{\alpha}, \tilde{a}_{\alpha}$ given by

$$
\begin{aligned}
& \tilde{\varphi}_{1}=-\frac{1}{\lambda_{p}} \sum_{j=1}^{p-1} \frac{1}{\lambda_{j}}\left\langle B_{\alpha}^{p j} B_{\alpha}^{j p}\right\rangle \\
& \tilde{\varphi}_{2}=-\frac{1}{\lambda_{p}} \sum_{j=1}^{p-1} \frac{1}{\lambda_{j}}\left\langle B_{\alpha}^{p j} B_{\alpha}^{j p+1}\right\rangle \\
& \tilde{\varphi}_{3}=-\frac{1}{\lambda_{p}} \sum_{j=1}^{p-1} \frac{1}{\lambda_{j}}\left\langle B_{\alpha}^{j p} B_{\alpha}^{p+1 j}\right\rangle \\
& \tilde{\varphi}_{4}=-\frac{1}{\lambda_{p}} \sum_{j=1}^{p-1} \frac{1}{\lambda_{j}}\left\langle B_{\alpha}^{p+1 j} B_{\alpha}^{j p+1}\right\rangle \\
& \tilde{a}_{\alpha}=\frac{B_{\alpha}^{p, p}}{\lambda_{p}} ; \quad \tilde{b}_{\alpha}=\frac{B_{\alpha}^{p, p+1}}{\lambda_{p}} ; \quad \tilde{c}_{\alpha}=\frac{B_{\alpha}^{p+1, p}}{\lambda_{p}} ; \quad \tilde{d}_{\alpha}=\frac{B_{\alpha}^{p+1, p+1}}{\lambda_{p}}
\end{aligned}
$$

Let us explain this result. 
In order to get $\gamma_{1}+\cdots+\gamma_{p}$, one can consider $p$ vectors $V_{\alpha}^{1} \cdots V_{\alpha}^{p}$ defined by the following recursion relations:

$$
\begin{aligned}
V_{\alpha+1}^{1}= & M_{\alpha} V_{\alpha}^{1} \\
V_{\alpha+1}^{2} & =M_{\alpha} V_{\alpha}^{2}-\left(\frac{\left(M_{\alpha} \cdot V_{\alpha}^{2}\right) \cdot V_{\alpha+1}^{1}}{V_{\alpha+1}^{1} \cdot V_{\alpha+1}^{1}}\right) V_{\alpha+1}^{1} \\
\vdots & \\
V_{\alpha+1}^{p} & =M_{\alpha} V_{\alpha}^{p}-\sum_{j=1}^{p-1}\left(\frac{\left(M_{\alpha} \cdot V_{\alpha}^{p}\right) \cdot V_{\alpha+1}^{j}}{V_{\alpha+1}^{j} \cdot V_{\alpha+1}^{j}}\right) V_{\alpha+1}^{j}
\end{aligned}
$$

By construction, for any $\alpha$, the vectors $V_{\alpha}^{1} \cdots V_{\alpha}^{p}$ are orthogonal and for $\mu$ small we can write them as

and

$$
V_{\alpha}^{j}=v_{\alpha}^{j}\left(\begin{array}{c}
\mu z_{1}^{j}(\alpha) \\
\cdot \\
\mu z_{j-1}^{j}(\alpha) \\
1 \\
\mu z_{j+1}^{j}(\alpha) \\
\cdot \\
\mu z_{n}^{j}(\alpha)
\end{array}\right) \quad \text { for } j \leqslant p-1
$$

$$
V_{\alpha}^{p}=v_{\alpha}^{p}\left[\begin{array}{c}
\mu t_{1}(\alpha) \\
\cdot \\
\mu t_{p-1}(\alpha) \\
x(\alpha) \\
y(\alpha) \\
\mu t_{p+2}(\alpha) \\
\cdot \\
\mu t_{n}(\alpha)
\end{array}\right]
$$

The multiplicative constants $v_{x}^{j}$ are normalization factors, which allow us to keep the $j$ th component of the vector $V_{x}^{j}$ normalized for $1 \leqslant j \leqslant p-1$ and to keep $x^{2}(\alpha)+y^{2}(\alpha)=1$.

Because all the eigenvalues have different moduli except the $p$ th and the $(p+1)$ th ones, one knows that in the limit $\mu \rightarrow 0$ each vector $V_{x}^{j}$, 
$j \leqslant p-1$, becomes an eigenvector of matrix $A$, whereas $V_{\alpha}^{p}$ belongs to the degenerate subspace associated with the eigenvalue $\lambda_{p}$.

Moreover, since they are orthogonal, we have

$$
\begin{gathered}
z_{j}^{i}(\alpha)+z_{i}^{j}(\alpha)=0 \quad \forall i, j \leqslant p-1 \\
t_{i}(\alpha)+x(\alpha) z_{p}^{i}(\alpha)+y(\alpha) z_{p+1}^{i}(\alpha)=0 \quad \forall i \leqslant p-1
\end{gathered}
$$

Then the $p$ th Liapunov exponent is given by

$$
\gamma_{p}=\lim _{N \rightarrow \infty} \frac{1}{2 N} \sum_{\alpha=1}^{N} \log \frac{\mathbf{V}_{\alpha+1}^{p} \cdot \mathbf{V}_{\alpha+1}^{p}}{\mathbf{V}_{\alpha}^{p} \cdot \mathbf{V}_{\alpha}^{p}}
$$

This method is very often used in the numerical determination of Liapunov exponents. ${ }^{(12,14)}$ From (77) we get the recurrence between the components of $V_{\alpha}^{j}$ and $V_{\alpha}^{p}$,

$$
\begin{aligned}
v_{\alpha+1}^{j} z_{p}^{j}(\alpha+1) & =\lambda_{p} v_{\alpha}^{j} z_{p}^{j}(\alpha)+B_{\alpha}^{p j} v_{\alpha}^{j}+O(\mu) \\
v_{\alpha+1}^{j} z_{p+1}^{j}(\alpha+1) & =\lambda_{p} v_{\alpha}^{j} z_{p+1}^{j}(\alpha)+B_{\alpha}^{p+1 j_{\alpha}}+O(\mu) \\
v_{\alpha+1}^{p} t_{j}(\alpha+1) & =\lambda_{j} v_{\alpha}^{p} t_{j}(\alpha)+B_{\alpha}^{j p} v_{\alpha}^{p} x(\alpha)+B_{\alpha}^{j p+1} v_{\alpha}^{p}(\alpha)+O(\mu)
\end{aligned}
$$

Using these relations, we also get

$$
\begin{aligned}
v_{\alpha+1}^{p} x( & +1) \\
= & \lambda_{p} v_{\alpha}^{p} x(\alpha)+\mu\left[B_{\alpha}^{p p} v_{\alpha}^{p} x(\alpha)+B_{\alpha}^{p p+1} v_{\alpha}^{p} y(\alpha)\right] \\
& -\mu^{2} \sum_{j=1}^{p-1} \frac{1}{v_{\alpha+1}^{j^{2}}}\left\langle\left[\lambda_{p} v_{\alpha}^{j} z_{p}^{j}(\alpha)+B_{\alpha}^{p j} v_{\alpha}^{j}\right]\right. \\
& \times\left\{\lambda_{p} v_{\alpha}^{p} x(\alpha)\left[\lambda_{p} v_{\alpha}^{j} z_{p}^{j}(\alpha)+B_{\alpha}^{p j} v_{\alpha}^{j}\right]\right. \\
& +\lambda_{p} v_{\alpha}^{p} y(\alpha)\left[\lambda_{p} v_{\alpha}^{j} z_{p+1}^{j}(\alpha)+B_{\alpha}^{p+1} v_{\alpha}^{j}\right] \\
& \left.\left.+\left[\lambda_{j} v_{\alpha}^{p} t_{j}(\alpha)+B_{\alpha}^{j p} v_{\alpha}^{p} x(\alpha)+B_{\alpha}^{j p+1} v_{\alpha}^{p} y(\alpha)\right] \cdot v_{\alpha+1}^{j}\right\}\right\rangle \\
& +O\left(\mu^{3}\right) \\
v_{\alpha+1}^{p} y(\alpha & +1) \\
= & \lambda_{p} v_{\alpha}^{p} y(\alpha)+\mu\left[B_{\alpha}^{p+1 p+1} v_{\alpha}^{p} y(\alpha)+B_{\alpha}^{p+1 p_{\alpha}} v_{\alpha}^{p} x(\alpha)\right] \\
& -\mu^{2} \sum_{j=1}^{p-1} \frac{1}{v_{\alpha}^{j^{2}}}\left\langle\left[\lambda_{p} v_{\alpha}^{j} z_{p+1}^{j}(\alpha)+B_{\alpha}^{p+1 j} v_{\alpha}^{j}\right]\right. \\
& \times\left\{\lambda_{p} v_{\alpha}^{p} x(\alpha)\left[\lambda_{p} v_{\alpha}^{j} z_{p}^{j}(\alpha)+B_{\alpha}^{p j} v_{\alpha}^{j}\right]\right. \\
& +\lambda_{p} v_{\alpha}^{p} y(\alpha)\left[\lambda_{p} v_{\alpha}^{j} z_{p+1}^{j}(\alpha)+B_{\alpha}^{p+1 j_{\alpha}} v_{\alpha}^{j}\right] \\
& \left.\left.+\left[\lambda_{j} v_{\alpha}^{p} t_{j}(\alpha)+B_{\alpha}^{j p} v_{\alpha}^{p} x(\alpha)+B_{\alpha}^{j p+1} v_{\alpha}^{p} y(\alpha)\right] \cdot v_{\alpha+1}^{j}\right\}\right\rangle \\
& +O\left\langle\mu^{3}\right\rangle
\end{aligned}
$$


The leading order in $\mu$ gives

$$
v_{\alpha+1}^{j}=\lambda_{j} v_{\alpha}^{j}+O(\mu) \quad \text { for } \quad 1 \leqslant j \leqslant p
$$

Therefore, one can replace $v_{x+1}^{j} / v_{\alpha}^{j}$ by $\lambda_{j}$ for $j \leqslant p-1$ in Eqs. (83) and (84) and in the term of order $\mu^{2}$ in (86), and one also can replace $v_{\alpha+1}^{p} / v_{\alpha}^{p}$ by $\lambda_{p}$ in Eq. (85). We have averaged the terms of order $\mu^{2}$ in (86) and (87) and neglected the fluctuations, which would contribute to higher orders in $\mu$.

Since the random $M(\alpha)$ are statistically independent, it is clear that

$$
\left\langle B_{\alpha}^{k, l} z_{i}^{j}(\alpha)\right\rangle=0
$$

for all indices $i, j, k, l$.

Moreover, (83) and (84) give us, at the lowest order in $\mu$,

$$
\begin{aligned}
\left\langle z_{p}^{j}(\alpha)^{2}\right\rangle & =\frac{1}{\lambda_{j}^{2}-\lambda_{p}^{2}}\left\langle\left(B_{\alpha}^{p, j}\right)^{2}\right\rangle \\
\left\langle z_{p+1}^{j}(\alpha)^{2}\right\rangle & =\frac{1}{\lambda_{j}^{2}-\lambda_{p}^{2}}\left\langle\left(B_{\alpha}^{p+1, j}\right)^{2}\right\rangle \\
\left\langle z_{p}^{j}(\alpha) z_{p+1}^{j}(\alpha)\right\rangle & =\frac{1}{\lambda_{j}^{2}-\lambda_{p}^{2}}\left\langle B_{\alpha}^{p, j} B_{\alpha}^{p+1, j}\right\rangle
\end{aligned}
$$

We can rewrite $v_{\alpha+1}^{p} x(\alpha+1)$ and $v_{\alpha+1}^{p} y(\alpha+1)$,

$$
\begin{aligned}
v_{\alpha+1}^{p} x(\alpha+1)= & \lambda_{p} v_{\alpha}^{p}\left[x(\alpha)+\mu\left(\tilde{a}_{\alpha} x(\alpha)+\tilde{b}_{\alpha} y(\alpha)\right)\right. \\
& \left.+\mu^{2}\left(\tilde{\varphi}_{1} x(\alpha)+\tilde{\varphi}_{2} y(\alpha)\right)\right]
\end{aligned}
$$

and

$$
\begin{aligned}
v_{\alpha+1}^{p} y(\alpha+1)= & \lambda_{p} v_{\alpha}^{p}\left[y(\alpha)+\mu\left(\tilde{c}_{\alpha} x(\alpha)+\tilde{d}_{\alpha} y(\alpha)\right)\right. \\
& \left.+\mu^{2}\left(\tilde{\varphi}_{3} x(\alpha)+\tilde{\varphi}_{4} y(\alpha)\right)\right]
\end{aligned}
$$

where the coefficients $\tilde{a}_{\alpha}, \tilde{b}_{x}, \tilde{c}_{\alpha}, \tilde{d}_{\alpha}$ and the $\tilde{\varphi}_{i}$ are given by (76).

Thus, we see that if we define $R^{\prime}$ and $R$ by

$$
\begin{aligned}
R^{\prime} & =v_{\alpha+1}^{p} x(\alpha+1) / v_{\alpha+1}^{p} y(\alpha+1) \\
R & =v_{\alpha}^{p} x(\alpha) / v_{\alpha}^{p} y(\alpha)
\end{aligned}
$$

one has

$$
R^{\prime}=\frac{R+\mu\left(\tilde{a}_{\alpha} R+\tilde{b}_{\alpha}\right)+\mu^{2}\left(\tilde{\varphi}_{1} R+\tilde{\varphi}_{2}\right)}{1+\mu\left(\tilde{c}_{\alpha} R+\tilde{d}_{\alpha}\right)+\mu^{2}\left(\tilde{\varphi}_{3} R+\tilde{\varphi}_{4}\right)}
$$


Using (82) and (97), we can extend the result (24) of Section 2 to the calculation of $\gamma_{p}$ :

$$
\begin{aligned}
& \gamma_{p}=\lim _{N \rightarrow+\infty} \frac{1}{N} \log \left|\frac{v_{N}^{p}}{v_{0}^{p}}\right| \\
& \gamma_{p}=\lim _{N \rightarrow+\infty} \frac{1}{2 N} \sum_{\alpha=1}^{N} \log \left(\frac{\nu_{\alpha+1}^{p}}{v_{\alpha}^{p}}\right)^{2}
\end{aligned}
$$

This can be calculated by taking the sum of the squares of Eqs. (93) and (94) and using the fact that $x(\alpha)^{2}+y(\alpha)^{2}=1$. This gives

$$
\begin{aligned}
\gamma_{p}= & \log \lambda_{p}+\frac{1}{2} \int P(R) d R\left\langle\operatorname { l o g } \left\{\left[\left(1+\mu \tilde{a}_{\alpha}+\mu^{2} \tilde{\varphi}_{1}\right) R+\mu \tilde{b}_{\alpha}+\mu^{2} \tilde{\varphi}_{2}\right]^{2}\right.\right. \\
& \left.\left.+\left[\left(\mu \tilde{c}_{\alpha}+\mu^{2} \tilde{\varphi}_{3}\right) R+1+\mu \tilde{d}_{\alpha}+\mu^{2} \tilde{\varphi}_{4}\right]^{2}\right\}-\log \left(R^{2}+1\right)\right\rangle / \int P(R) d R
\end{aligned}
$$

We recognize the same expression as in Section 2 [compare (22) and (97), (24) and (100)]. Therefore $\gamma_{p}$ can be expanded for $\mu$ small as

$$
\gamma_{p}=\log \lambda_{p}+\frac{1}{2} \mu^{2} \int_{-\infty}^{+\infty} P_{0}(R) G(R) d R / \int_{-\infty}^{+\infty} P_{0}(R) d R
$$

where $P_{0}(R)$ and $G(R)$ are still given by formulas (13) and (12), where the $\varphi_{i}$ and $a_{\alpha}, b_{\alpha}, c_{\alpha}, d_{\alpha}$ are replaced by the $\tilde{\varphi}_{i}$ and $\tilde{a}_{\alpha}, \tilde{b}_{\alpha}, \tilde{c}_{\alpha}, \tilde{d}_{\alpha}$.

\section{CONCLUSION}

In this work we have developed a method which gives the weak disorder expansion of the Liapunov exponents when the matrix $A$ has two degenerate eigenvalues. It would be interesting to generalize this calculation to many cases. (1) a degeneracy larger than 2; (2) nondiagonalizable matrices; (3) the case where matrix $A$ has several complex eigenvalues with the same modulus.

In principle the main result (11)-(15) of the present work can be compared with the nondegenerate expansion (5). Consider the case where the matrix $A$ has the form

$$
A=\left(\begin{array}{cc}
1+\mu^{2} \varphi_{1} & 0 \\
0 & 1+\mu^{2} \varphi_{4}
\end{array}\right)
$$


when the difference $\varphi_{1}-\varphi_{4}$ becomes large

$$
\Delta \varphi=\varphi_{1}-\varphi_{4} \rightarrow \infty
$$

In that limit the two eigenvalues of matrix $A$ can be considered as nondegenerate and therefore we can use expression (5). The term of order $\mu^{4}$ gives the $1 / \Delta \varphi$ correction, and so from (5) one gets in the limit $\Delta \varphi \rightarrow \infty$

$$
\gamma_{1}=\mu^{2}\left[\varphi_{1}-\frac{\left\langle a_{\alpha}^{2}\right\rangle}{2}+\frac{1}{\Delta \varphi}\left(\left\langle a_{\alpha} b_{\alpha}\right\rangle\left\langle a_{\alpha} c_{\alpha}\right\rangle-\frac{\left\langle b_{\alpha}^{2}\right\rangle\left\langle c_{\alpha}^{2}\right\rangle}{4}\right)+O\left(\frac{1}{(\Delta \varphi)^{2}}\right)\right]
$$

We tried to see whether this result could be recovered from expressions (11)-(15) in the limit $\Delta \varphi \rightarrow \infty$, but we have not succeeded in proving or disproving it. We could only compute $\gamma_{1}$ in some examples; for example, cases when

$$
\lim _{A \rightarrow \infty} \int_{-A}^{A} d x \frac{H_{2}(x)}{H_{1}(x)}=0
$$

and in all the cases we could solve, we found an agreement between the degenerate expansion (11)-(15) and the nondegenerate expansion (5).

To conclude, we mention that the problem of the sensitivity of the Liapunov exponents of products of random matrices to small perturbations has been discussed in the mathematics literature ${ }^{(15-18)}$ and that there exist conditions under which Liapunov exponents are smooth functions of the perturbation.

\section{ACKNOWLEDGMENTS}

We have benefited from many discussions with J.L. Pichard. We thank a referee for pointing out Refs. 15-18.

\section{REFERENCES}

1. F. J. Dyson, Phys. Rev. 92:1331 (1953).

2. S. Alexander, J. Bernasconi, W. R. Schneider, and R. Orbach, Rev. Mod. Phys. 53:175 (1981).

3. B. Derrida, K. Mecheri, and J. L. Pichard, J. Phys. (Paris) 48:733 (1987).

4. J. P. Eckmann and D. Ruelle, Rev. Mod. Phys. 57:617 (1985).

5. R. Livi, A. Politi, S. Ruffo, and A. Vulpiani, J. Stat. Phys. 46:147 (1987), and references therein.

6. J. P. Bouchaud, A. Georges, and P. Le Doussal, Lecture given at the Meeting on Dynamical Systems, Rome 1986, and references therein. 
7. P. Bougerol and J. Lacroix, Products of Random Matrices with Applications to Schrödinger Operators (Birkhaüser, 1985).

8. C. M. Newman, Commun. Math. Phys. 103:121 (1986).

9. B. Derrida, M. Mendès France, and J. Peyrière, J. Stat. Phys. 45:439 (1986).

10. I. S. Gradshteyn and I. M. Ryzhik, Table of Integrals, Series and Products (Academic Press, New York, 1980), p. 262.

11. C. M. Newman, Contemp. Math. 50:121 (1986).

12. J. L. Pichard and G. Sarma, J. Phys. C 14:L127 (1981).

13. J. L. Pichard and G. Sarma, J. Phys. C 14:L617 (1981).

14. G. Benettin, L. Galgani, A. Giorgilli, and J. M. Strelcyn, C. R. Acad. Sci. Paris 286A:431 (1978).

15. Y. Kifer and E. Slud, Ergod. Theory Dynam. Syst. 2:367 (1982).

16. Y. Kifer, Z. Wahr. 61:83 (1982).

17. H. Furstenberg and Y. Kifer, Israel J. Math. 46:12 (1983).

18. H. Hennion, Z. Wahr. 67:265 (1984).

19. B. Derrida and E. Gardner, J. Phys. (Paris) 45:1283 (1984).

20. J. P. Bouchaud, A. Georges, D. Hansel, P. Le Doussal, and J. M. Maillard, J. Phys. A 19:L1145 (1986). 